\title{
Power System Harmonics Estimation using Signal Processing Techniques
}

\author{
Pratap Sekhar Puhan ${ }^{1}$, Pravat Kumar Ray ${ }^{2}$ and Gayadhar Panda ${ }^{3}$ \\ ${ }^{1}$ Department of Electrical Engineering, BIET Bhadrak \\ ${ }^{2}$ Department of Electrical Engineering, NIT Rourkela \\ ${ }^{3}$ Department of Electrical and Electronics Engineering, NIT Meghalaya
}

\begin{abstract}
This paper proves the effectiveness of Least Mean Square (LMS) estimation algorithm for estimation of power system harmonics, inter-harmonics and sub-harmonics of distorted signal. Accurate estimation can be achieved by this technique for harmonics, sub-harmonics and inter-harmonics estimation including possible variation in the signal such as change in amplitude when applied to a distorted power system signal. This technique is compared with Least square based algorithm and Recursive least square (RLS) Algorithm simulation and experimental studies are carried out for the above three algorithm and it is observed that the proposed LMS approach to estimation of harmonics, inter-harmonics and sub-harmonics in a distorted power system signal exhibits superior estimation performance in terms of tracking time and accuracy as compared to performances Least Square based algorithm i.e Recursive Least Square (RLS) algorithm.
\end{abstract}

Keywords: Fast Fourier Transform (FFT), Harmonics Estimation, Least Mean Square (LMS), Recursive Least Square (RLS).

\section{Introduction}

Development of power semiconductor technology, power electronics based devices such as static var compensators, adjustable speed drives and uninterruptible power supplies are widely employed in various applications results, Non-linear characteristics of voltage and current which indicates the degradation of power quality, these devices draw current with harmonic content and reactive power from $\mathrm{AC}$ mains. Both harmonics and inter-harmonics have quite negative impacts on power networks and customers. Negative effects of harmonics are increased $I^{2} R$ losses, over voltage, unbalancing and mal-operations of the relays and saturation of transformer core. Thus, harmonic estimation is one of the critical and challenging issues while dealing with power system signals.

Recent literature [1-11] presents different techniques on power system harmonics estimation. In order to get the voltage and current frequency spectrum from discrete time samples, most frequency domain harmonic analysis algorithms are based on the Fast Fourier transform (FFT). However, FFT [3] based technique suffers from leakage effect i.e an effect in the frequency analysis of finite length signals or finite length segments of infinite signals where it appears as if some energy has 'leaked' out of the original signal spectrum into other frequencies and its performance highly degrades while estimating inter and sub-harmonics, including frequency deviations. Kalman Filter $[8,14]$ is one of the robust algorithms for estimating the magnitudes of sinusoids of known frequencies embedded in an unknown measurement noise. But this algorithm fails to track any dynamic changes of signal such as sudden changes in amplitude, phase or frequency of signal. RLS is one of the method for estimation of harmonics, but main difficulty lies in initial choice of Co-variance matrix and similarly Adaline [12-13] can be used for harmonics estimation but its demerit lies on initial choice of weight vector. To overcome the problems faced in the said techniques, Least Mean Square (LMS) approach is considered in this paper for harmonics estimation.

The paper is organized as follows. Section II describes the harmonics estimation techniques. Section III presents simulation studies on one existing method such as RLS [15-16] together with the proposed LMS approach for harmonics estimation applied to distorted power signals. Section IV describes the experimental setup developed to validate the efficacy of the proposed algorithm. Section V concludes the paper.

A. Least Mean Square (LMS) based harmonic estimation

\section{Methods Of Harmonics Estimation}

Let us assume the voltage or current waveforms of the known fundamental angular frequency $\omega$ as the sum of harmonics of unknown magnitudes and phases. The general form of the waveform is

$$
y(t)=\sum_{n=1}^{N} A_{n} \sin \left(\omega_{n} t+\phi_{n}\right)+A_{d c} \exp \left(-\alpha_{d c} t\right)+\varepsilon(t)
$$


where $N$ is the number of harmonics. $\omega_{n}=n 2 \pi f_{0} ; f_{0}$ is the fundamental frequency; $\varepsilon(t)$ is the additive noise; $A_{d c} \exp \left(-\alpha_{d c} t\right)$ is the dc offset decaying term.

After discretization of Eq. (1) with a sampling period, $T$ one obtains the following expressions

$y(k)=\sum_{n=1}^{N} A_{n} \sin \left(\omega_{n} k T+\phi_{n}\right)+A_{d c} \exp \left(-\alpha_{d c} k T\right)+\varepsilon(k)$

Invoking Taylor series expansion of the dc decaying term, $A_{d c} \exp \left(-\alpha_{d c} t\right)$ and retaining only first two terms of the series yields

$y_{d c}=A_{d c}-A_{d c} \alpha_{d c} k T$

Using Eq. (3) in Eq. (2), $y(k)$ can be obtained as

$y(k)=\sum_{n=1}^{N} A_{n} \sin \left(\omega_{n} k T+\phi_{n}\right)+A_{d c}-A_{d c} \alpha_{d c} k T+\varepsilon(k)$

For estimation amplitudes and phases Eq.(4.4) can be rewritten as

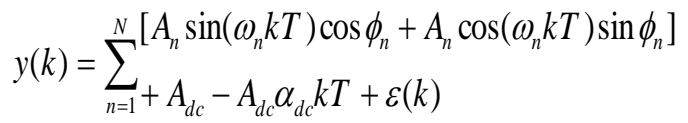

Eq. (5) can be rewritten in parametric form as follows

$y(k)=H(k) X$

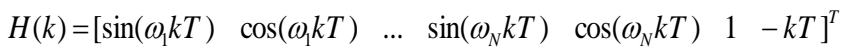

(6)

The vector of unknown parameter

$X(k)=\left[\begin{array}{lllllll}X_{1}(k) & X_{2}(k) & \ldots & X_{2 N-1}(k) & X_{2 N}(k) & X_{2 N+1}(k) & X_{2 N+2}(k)\end{array}\right]^{T}$

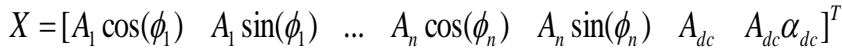

(8)

The LMS algorithm is applied to estimate the state. The algorithm minimizes the square of the error recursively by altering the unknown parameter $X_{k}$ at each sampling instant using equation (9) given below

$X_{k}=X_{k-1}+\mu e_{k} y_{k}^{\wedge}$

where the error signal is

$e_{k}=y_{k}-\hat{y_{k}}$

After the updating of the vector of unknown parameter using LMS algorithm, amplitudes, phases of the fundamental and nth harmonic parameters and dc decaying parameters are derived as

$$
\begin{aligned}
& A_{n}=\sqrt{\left(X_{2 N}^{2}+X_{2 N-1}^{2}\right)} \\
& \phi_{n}=\tan ^{-1}\left(\frac{X_{2 N}}{X_{2 N-1}}\right) \\
& A_{d c}=X_{2 N+1} \\
& \alpha_{d c}=\left(\frac{X_{2 N+2}}{X_{2 N+1}}\right)
\end{aligned}
$$

B. Recursive Least Square (RLS) based harmonic estimation

The signal as described in section II.A is taken; the vector of unknown parameter $X$, as in (7) is updated using RLS as

$X$ is updated using Recursive Least Square Algorithm as

$\hat{X}(k+1)=\hat{X}(k)+K(k+1) e(k+1)$

Error in measurement is

$e(k+1)=y(k+1)-H(k+1)^{T} \hat{X}(k)$ 
The gain $\mathrm{K}$ is related with covariance of parameter vector

$$
K(k+1)=P(k) H(k+1)\left[1+H(k+1)^{T} P(k) H(k+1)\right]^{-1}
$$

The updated covariance of parameter vector using matrix inversion lemma

$$
P(k+1)=\left[I-K(k+1) H(k+1)^{T}\right] P(k)
$$

These equations are initialized by taking some initial values for the estimate at instants $t, \theta(t)$ and $P$. As the choice of initial covariance matrix is large it is taken as $P=\alpha I$, where $\alpha$ is a large number and $I$ is a square identity matrix.

After the updating of the vector of unknown parameters using Recursive Least Square (RLS) algorithm, amplitudes, phases of the fundamental and nth harmonic parameters and dc decaying parameters can be derived using (10)-(13)

\section{Simulation Results}

\section{A. Static signal corrupted with random noise and decaying DC component}

To evaluate the performance of the proposed algorithm in estimating harmonics amplitude and phase, Numerical experiments implemented in MATLAB have been performed. The power system signal used for the estimation, besides the fundamental frequency, contains higher harmonics of the 3rd, 5th, 7th, 11th and a slowly decaying DC component. This kind of signal is typical in industrial load comprising power electronic converters and arc furnaces [4].

$$
\begin{aligned}
& y(t)=1.5 \sin \left(\omega t+80^{\circ}\right)+0.5 \sin \left(3 \omega t+60^{\circ}\right)+0.2 \sin \left(5 \omega t+45^{0}\right) \\
& +0.15 \sin \left(7 \omega t+36^{\circ}\right)+0.1 \sin \left(11 \omega t+30^{\circ}\right)+0.5 \exp (-5 t)+\varepsilon(t)
\end{aligned}
$$

The signal is corrupted by random noise $\varepsilon(t)=0.05$ randn of zero mean, normal distribution and unity variance. In the simulation, using LMS, initial values of $\mu=0.001$ and $W=0.018$ are taken. In case of RLS, the value of $\delta$ is taken as 100 .

Fig. 1 shows actual vs. estimated value of signal using RLS and LMS algorithms. Actual vs estimated signal almost matches with each other with little deviation in case of RLS algorithm. Fig. 2-5 show the tracking of fundamental and 3rd harmonics signal components in presence of random noise and decaying dc components using RLS and LMS, algorithms. RLS algorithm is tuned optimally by properly choosing the co-variance and noise variance matrices. Using RLS, time required to reach the actual values for the fundamental and harmonics components is 0.02 seconds or more on a $50 \mathrm{~Hz}$ system. Using LMS, the exact value of fundamental and harmonics components are obtained roughly in $0.01 \mathrm{sec}$. or less than that based on a $50 \mathrm{~Hz}$ waveform in presence of random noise and decaying dc components. From these Figures, it is found that performance of estimation using LMS is better than RLS

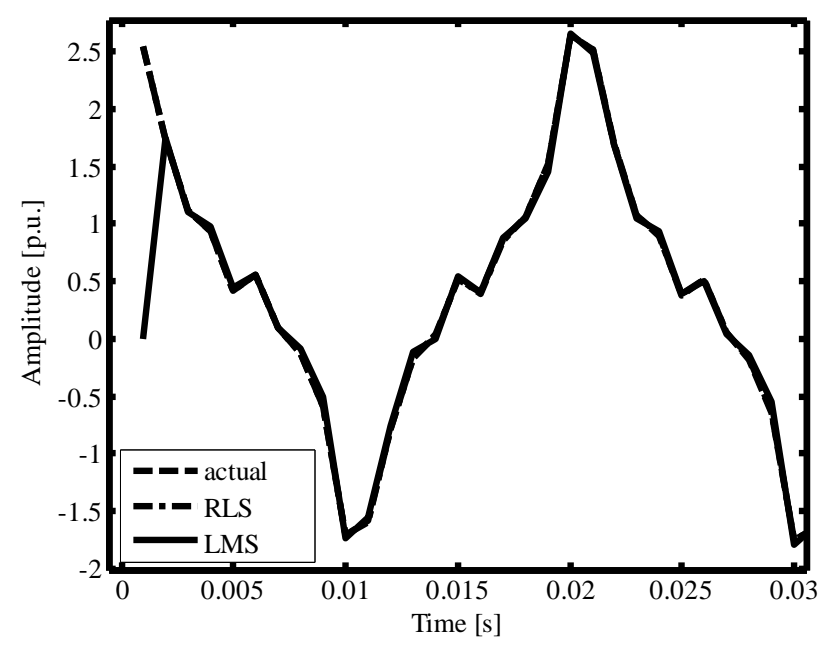

Fig.1. Actual and Estimated signal using RLS and LMS 


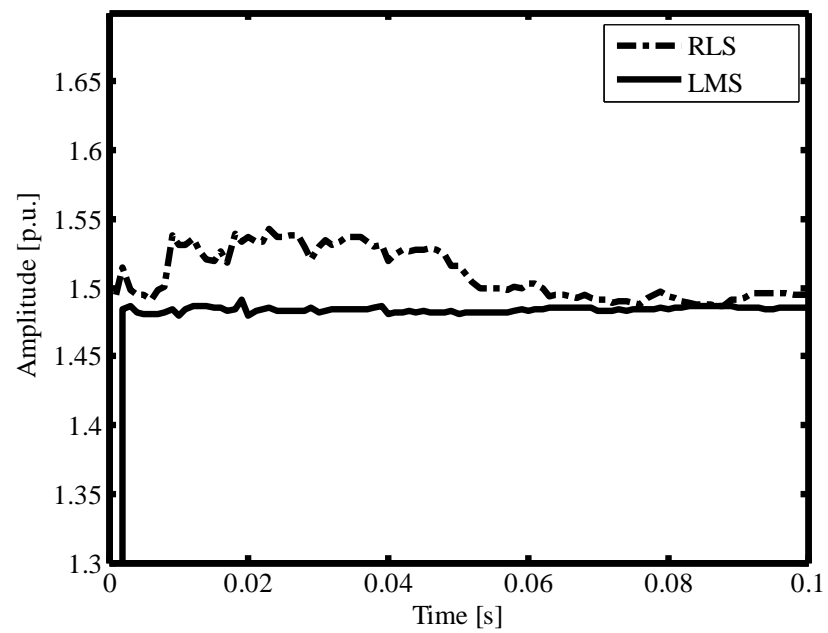

Fig.2. Estimation of Fundamental Amplitude of signal

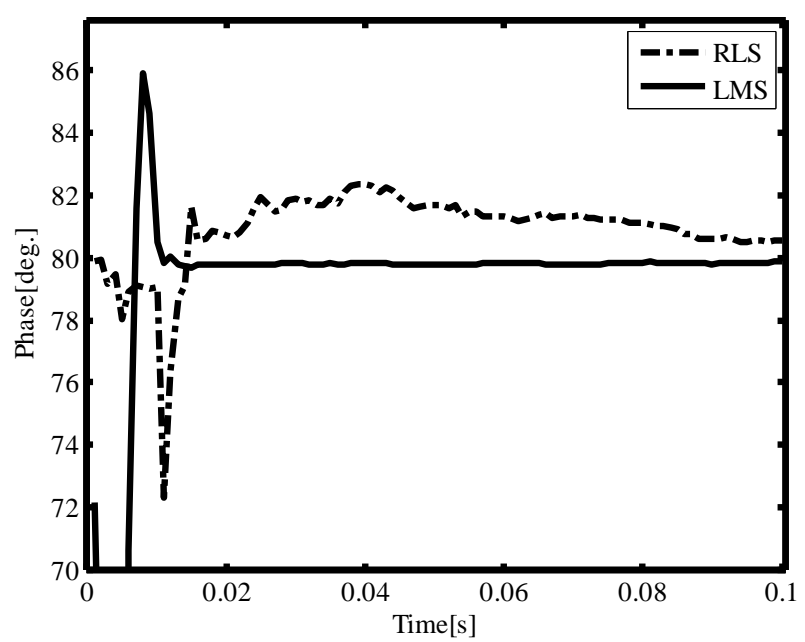

Fig.3. Estimation of Phase of Fundamental component of signal

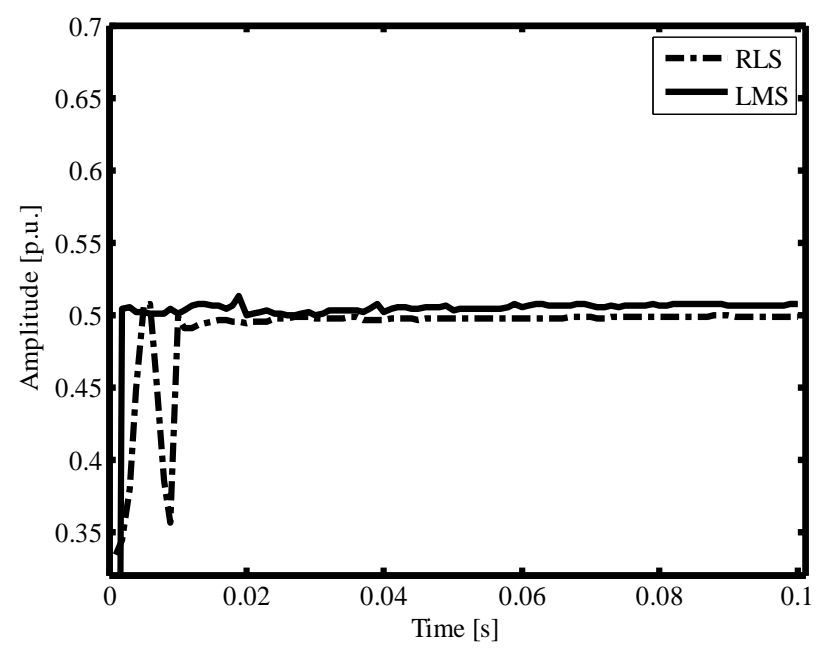

Fig.4. Estimation of amplitude of $3^{\text {rd }}$ harmonic component of signal 


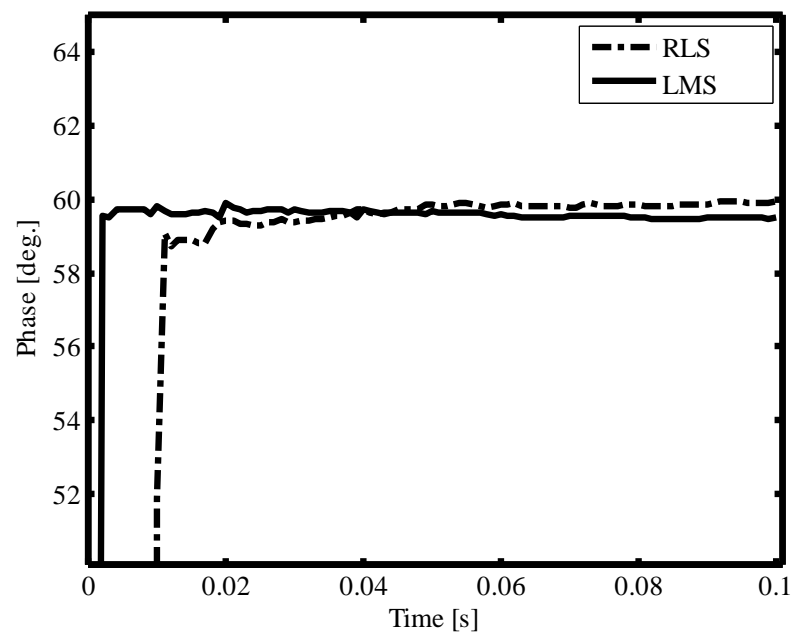

Fig.5. Estimation of Phase of $3^{\text {rd }}$ Harmonic component of signal

\section{B. Estimation of harmonics in presence of amplitude drift}

For considering the real time situations of a power system signal, abrupt change in amplitude of signal is taken into account in this section. Case of $3^{\text {rd }}$ harmonic component change is considered and is allowed to change from 0.5 p.u. to 2 p.u. at 0.05 second. Proposed algorithm takes $1 \mathrm{~ms}$ to reach steady state during transition, that can be marked in the zoomed figure.

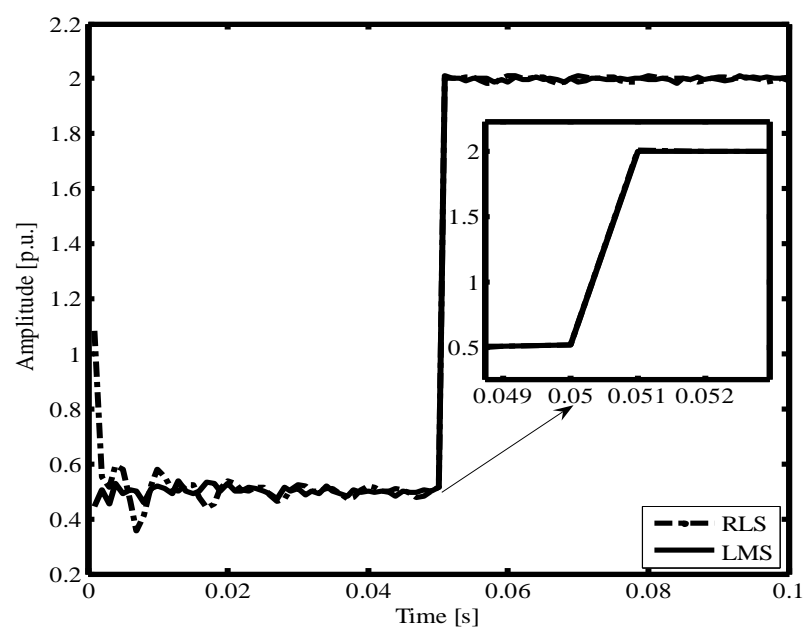

Fig.6. Estimation of $3^{\text {rd }}$ Harmonic amplitude during amplitude drift

C. Harmonics estimation of signal in presence of inter and sub-harmonics

To evaluate the performance of the proposed algorithm in the estimation of a signal in the presence of subharmonics and inter-harmonics, a sub-harmonic and two inter-harmonics components are added to the original signal as given in (4.28). 


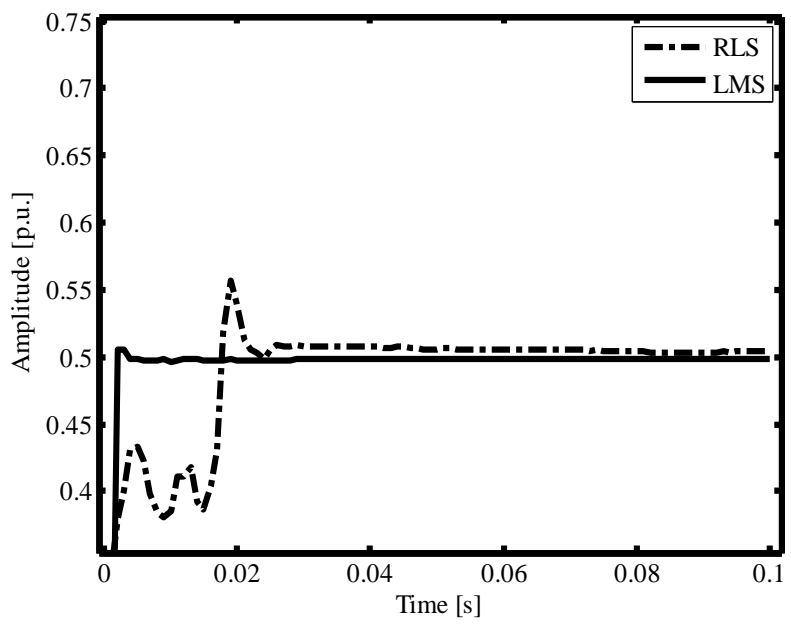

Fig.7. Estimation of amplitude of sub-harmonic component of signal

The frequency of sub-harmonic is $20 \mathrm{~Hz}$, the amplitude is set to be 0.5 p.u. and the phase is equal to 75 degrees. The frequency, amplitude and phase of one of the inter-harmonics are $130 \mathrm{~Hz}, 0.25$ p.u. and 65 degrees respectively. The frequency, amplitude and phase of the other inter-harmonic are $180 \mathrm{~Hz}, 0.35$ p.u. and 10 degrees respectively. Fig. 7 shows the amplitude of estimation of sub-harmonic component of signal and is found that comparatively better estimation is obtained in case of LMS

\section{Experimental Studies And Results}

In view of real-time application of the algorithm for estimating harmonics in a power system, voltage data generation was accomplished in the laboratory on running a DG set using the experimental setup as shown in Fig. 8.

Specifications of the Instruments used are:

1. D-G set:

a) Alternator-3 phase, $50 \mathrm{~Hz}$, Y connected, 415 volt, $1500 \mathrm{rpm}, 55.8 \mathrm{~A}, 40 \mathrm{KVA}$

b) Diesel Engine- Bore $\times$ stroke $=110 \times 116,37.2 \mathrm{KW}, 1500 \mathrm{rpm}$

2. Rheostats: $100 \mathrm{ohm}, 5 \mathrm{~A}$ (3 in no.)

3. Non-linear load: 3 Phase diode bridge rectifier with a $5 \Omega$ resistor in series with a $100 \mathrm{mH}$ inductor at the d.c side.

4. Digital Storage Oscilloscope: Band Width-200MHz, Sample rate-2GS/s, Channels-2, Record length-2500 data points, PC Connectivity- USB Port and Open Choice PC Communication software, Probe-P2220

5. PC: $1.46 \mathrm{GHz}$ CPU and 1GB RAM, Notebook PC

The input voltage waveform is stored in a Digital Storage Oscilloscope (Tektronix Ltd.) across almost 10-ohm resistance (measured using multi-meter) and then through Open Choice PC Communication software, data is acquired to the personal computer.

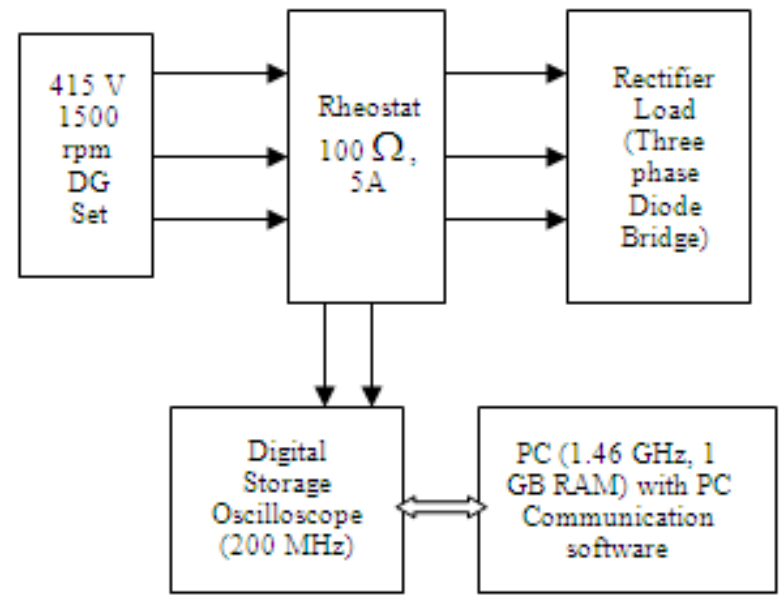

Fig.8. Experimental setup for online data generation 


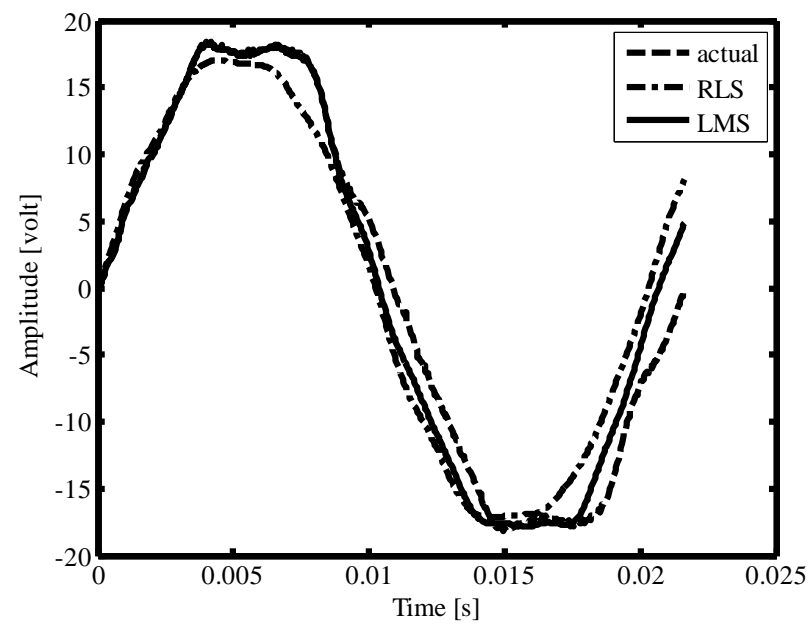

Fig.9. Estimation of signal from real data

Fig. 9 shows the estimation of voltage signal using RLS and LMS algorithms from the real data obtained from the experiment. In Fig. 9, the estimated waveform approaches the actual waveform over the cycle.

\section{Conclusions}

This paper presents a Least Mean Square (LMS) algorithm, for accurate estimation of amplitude and phase of the harmonic components of distorted power system signal. Improved harmonics estimation performance has been achieved by using the LMS algorithm as compared RLS algorithm. Due to less computational burden, this proposed LMS algorithm is suitable for online estimation. Several computer simulation tests have been conducted to estimate harmonics in a power system signal corrupted with random noise and decaying dc offsets. The simulation results including inter-harmonics and sub-harmonics with SNR 20 $\mathrm{dB}$ shows the robustness of the proposed LMS algorithm for effective harmonic estimation. For testing the effectiveness further, the same is tested for experimental data and found to provide satisfactory results for harmonic estimation.

\section{References}

[1] H. Mori, “An artificial neural network based method for power system voltage harmonics," IEEE Trans. Power Del., vol. 7, no.1, pp. 402-409, 1992.

[2] E.A.Abu, Al-Feilat, I.El.Amin, and M.Bettayeb, "Power system harmonic estimation a comparative study," Electric Power System Research, vol. 29, no.2, pp.91-97, 1994.

[3] Y.N.Chang, Y.C.Hsieh, C.S.Moo, "Truncation effects of FFT on estimation of dynamic harmonics in a power system," in Proc. 2000 Power System Technology, International Power Conf., vol.3, pp.1155-1160.

[4] P.K.Dash, D.P.Swain, A.Routray and A.C.Liew, "Harmonic estimation in a power system using adaptive perceptrons," IEE Proceedings Generations, Transmission and Distribution, vol. 143, no.6, pp. 565-574, 1996.

[5] Cai Tao, Duan Shanxu, Ren Ting and Liu Fangruli “ A robust parametric method for power harmonics estimation based on Mestimators" Measurement, vol.43, issue.1, pp. 67-77, January 2010

[6] Ahmet S. Yilmatz, Ahmed Alkan, Musa H. Asyali "Application of parametric spectral estimation methods on detection of power system harmonics” Electric Power System Research, Vol-78, pp. 683-693, 2008.

[7] T.Lobos,T.Kozina \& H.J.Koglin," "Power system harmonic estimation using Linear Least Square method \& SVD," IEE Proceedings Generations, Transmission and Distribution, vol. 148, no.6, pp. 567-572, 2001

[8] P.K.Dash and A.Sharaf, “A Kalman filtering approach for estimation of power system harmonics,” in Proc. 1998, $3{ }^{\text {rd }}$ Int. Conf. Harmonics Power System, Nashville, TN .

[9] N.R.Watson, J.Arrillaga, "Review Harmonics in large systems’ Electric Power System Research,” vol. 66, no.1, pp. 15-29, 2003.

[10] Karren Kennedy, Gordon Lightbody \& Robert Yacamini, "Power system harmonic analysis using the Kalman filter," in Proc. 2003 IEEE Power Engineering Society General Meeting, Toronto, Canada, vol.2, pp.752-757, 13-17 July 2003.

[11] Josif J. Tomic, Miodrag D. Kusljevic and Vladimir V. Vujicic "A new Power System Digital Harmonic analyzer" IEEE Transactions on Power Delivery, vol.22, no.2, pp.772-780, 2007

[12] M. Joorabian, S.S. Mortazavi, A.A. Khayyami " Harmonics estimation in a power system using a novel-hybrid Least Square Adaline algorithm” Electric Power System Research, vol.79, issue.1, pp. 107-116, 2009

[13] S. Ghodratollah Seifossadat, Morteza Razzaz, Mahmood Moghaddasian and Mehdi Monadi“ Harmonics Estimation in Power System Using Adaptive Perceptrons based on a Genetic Algorithm" WSEAS Trans. on Power Systems, vol.2, issue 11, 2007

[14] Chien-Hung Huang, Chien-Hsing Lee, Kuang-Jung Shih and Yaw-Juen Wang, “ Frequency Estimation of Distorted Power System Signals Using a Robust Algorithm,” IEEE Trans. on Power Delivery, vol.23, no.1, pp. 41-51, 2008

[15] Simon Haykin, Thomas Kailath, Adaptive Filter Theory, $4^{\text {th }}$ ed., Pearson Education, 2007.

[16] L. Jung, System Identification-Theory for the User, $2^{\text {nd }}$ ed., PTR Prentice Hall, Upper Saddle River, NJ, 1999. 